\title{
Condiciones de maceración y fermentación que incrementan el contenido de etanol en mosto fermentado para whisky de malta
}

\author{
Mashing and fermentation conditions that increase ethanol content in fermented wort \\ for single malt whisky
}

\begin{abstract}
María Teresa Rivera Jiménez ${ }^{1}$ Ramon Huerta Zurita ${ }^{2 *}$, María Guadalupe Herrera Hernández ${ }^{2}$, Violeta Herrera Enciso ${ }^{1}$, Carlos Alberto Flores Gómez ${ }^{1}$

Tecnológico Nacional de México, Campus Roque, Celaya, Guanajuato, México.

2 Instituto Nacional de Investigaciones Forestales, Agrícolas y Pecuarias, Campo Experimental Bajío, Celaya, Guanajuato, México.*rhzurita@yahoo.com.mx
\end{abstract}

\section{RESUMEN}

El contenido de etanol en mosto fermentado es el parámetro más importante para la producción de whisky en términos de volumen de destilado por unidad de malta. La biosíntesis de etanol durante la fermentación de mosto depende de muchos factores; no obstante, la cantidad inicial de carbohidratos fermentables afecta significativamente el contenido final de etanol en el mosto fermentado. El objetivo de este estudio fue evaluar el efecto de diferentes condiciones de maceración y fermentación sobre el contenido de etanol en mosto fermentado; asimismo, determinar las condiciones que contribuyen en el incremento de este compuesto. Se observó que la maceración isotérmica a $65^{\circ} \mathrm{C}$ por $3 \mathrm{~h}$, con una relación malta:agua 1:3, resulta en mostos con mayor contenido de carbohidratos fermentables. Además, se determinó que el mejor rendimiento de etanol se obtiene con fermentadores cerrados a $20^{\circ} \mathrm{C}$ en mostos de $12.5^{\circ} \mathrm{P}$. Lo anterior resulta en una eficiencia de $0.34 \mathrm{~g}$ de etanol $/ \mathrm{g}$ de sólidos solubles; además de la reducción significativa de metanol y glicerol.

Palabras clave: whisky, malta, maceración, fermentación, rendimiento de etanol.

\section{ABSTRACT}

Ethanol content in fermented wort is the most important parameter for whisky production, in terms of volume of distillate per unit of malt. Ethanol biosynthesis during wort fermentation depends on many factors, however, the initial concentration of fermentable carbohydrates significantly affects the final ethanol content in fermented wort. The objective of this study was to evaluate the effect of different mashing and fermentation conditions on ethanol content in fermented wort, and to determine the conditions that better contribute to the increase of this compound. It was observed that an isothermal mashing of $65^{\circ} \mathrm{C}$ for $3 \mathrm{~h}$, with a 1:3 grist:water ratio, results in worts with high fermentable carbohydrates content. In addition, it was determined that the higher ethanol yield is attained with closed fermenters at $20^{\circ} \mathrm{C}$ and $12.5{ }^{\circ} \mathrm{P}$ worts. These conditions result in a conversion efficiency of $0.34 \mathrm{~g}$ of ethanol $/ \mathrm{g}$ of soluble solids, in fermented worts with reduced amounts of methanol and glycerol.
Key words: whisky, malt, mashing, fermentation, ethanol yield.

\section{INTRODUCCIÓN}

Uno de los principales objetivos en la industria de bebidas fermentadas y destiladas es la generación eficiente de etanol debido a que este parámetro define el volumen final de producto obtenido por unidad de materia prima (Stewart, 2009; Nicol, 2014). Esto ha sido una constante en bebidas alcohólicas de mucha tradición como la cerveza y el whisky, mismas que comparten ciertas similitudes en cuanto a su procesamiento (Bathgate, 2016). A pesar de que ambas bebidas se pueden elaborar con otros granos como maíz, centeno, trigo y cebada no malteada; es necesaria la inclusión de una porción de malta de cebada en las recetas, o de otras fuentes de enzimas en caso de ser permitido, para la adecuada conversión del almidón en azúcares fermentables durante la maceración (Agu et al., 2006; Bathgate, 2016). Se ha reportado que la variación en las condiciones de maceración determina el rendimiento y composición del mosto; no obstante, la mayoría de estos reportes se han dado con fines cerveceros (Evans et al., 2005; Schwarz et al., 2007; Evans et al., 2010; Evans et al., 2011). Tanto en cervecería como en destilería se busca la obtención de mostos con alto contenido de azúcares fermentables; sin embargo, mientras que en cervecería se busca un balance adecuado entre azúcares fermentables y limite dextrinas debido a que éstas últimas contribuyen en el cuerpo de la cerveza, en destilería se busca maximizar el desdoblamiento del almidón para la mayor generación posible de etanol, parámetro comúnmente denominado 'spirit yield' (Lyons, 2003; Bathgate, 2016). Durante la fermentación, tanto la concentración de azúcares fermentables como de compuestos nitrogenados determinan en gran medida la eficiencia de la levadura en la síntesis de etanol (Stewart et al., 2013). Además, existen otros factores como la alta concentración de sólidos en mosto que incrementan la presión osmótica del medio y que afectan en consecuencia la estabilidad celular de las levaduras durante la fermentación (Nevoigt y Stahl, 1997; Cronwright et al., 2002). A pesar de las similitudes que comparten en algunas etapas de proceso la cervecería y la destilería, los mayores avances 
tecnológicos post fermentación se han dado en el ámbito cervecero debido a requerimientos de calidad de cerveza que se asocian a su estabilidad coloidal, aceptación y vida de anaquel (Sadosky et al., 2002; Jin et al., 2004; Miedl et al., 2005; Vanderhaegen et al., 2006). A diferencia de la cerveza, en bebidas destiladas como el whisky, muchos de los compuestos generados durante la fermentación permanecen en el residuo de destilación considerando que el principal objetivo de esta etapa del proceso es la eficiente recuperación y concentración de etanol (Nicol, 2014). Debido al hecho de que las etapas post fermentación no implican síntesis de etanol durante el procesamiento de whisky, las condiciones de maceración y fermentación se vuelven determinantes en la generación de este compuesto. Dada la amplia variabilidad de condiciones de maceración y fermentación en el procesamiento de whisky, tanto a nivel comercial como artesanal (Bringhurst y Brosnan, 2014; Fukuyo y Myojo, 2014; Lyons, 2014; Quinn, 2014), el objetivo de este trabajo fue estudiar las condiciones de proceso que afectan y contribuyen en el contenido final de etanol en mosto fermentado. De esta forma, se busca contribuir en la uniformidad de condiciones en etapas pre destilación para la eficiente generación de etanol en la producción de whisky de malta; especialmente en el nivel artesanal, donde el número de destilerías ha crecido sustancialmente en la última década.

\section{MATERIALES Y MÉTODOS}

Éste estudio constó de experimentos secuenciales donde el mejor tratamiento de cada experimento evaluado se utilizó como base para el experimento subsecuente. En esta sección sólo se describen los experimentos y sus tratamientos; mientras que, en la sección de resultados y discusión, se describen y discuten los resultados que sustentaron la toma de decisiones.

En todos los experimentos se utilizó una malta comercial elaborada a partir de cebada de dos hileras con las siguientes características de calidad determinadas en laboratorio conforme los métodos oficiales de la American Society of Brewing Chemists (ASBC, 2009): humedad, 5 \%; extracto molienda fina, $85 \%$ base seca (b.s.); extracto molienda gruesa, 84 \% b.s.; proteína total (PT), 11 \% b.s.; proteína soluble, $5.3 \%$ b.s.; alfa-amilasa, 65 unidades de dextrinificación a $20^{\circ} \mathrm{C}$ (U.D. $20^{\circ} \mathrm{C}$ ) b.s.; poder diastásico (DP), 135 grados ASBC ( $\left.{ }^{\circ} \mathrm{ASBC}\right)$ b.s.; friabilidad, $92 \%$; viscosidad de mosto, 1.52 centipoise (cP); amino nitrógeno libre (FAN), 200 ppm; y color de mosto, 2.0 grados del Standard Reference Method de la ASBC ('SRM).

Este estudio se dividió en dos fases. En la fase 1 se evaluaron diferentes tratamientos para determinar las mejores condiciones de maceración; mientras que en la fase 2 , se buscaron las mejores condiciones de fermentación. Para la molienda de malta en la fase 1 se utilizó un molino de discos para laboratorio Bühler DLFU (Bühler Group, Uzwil, Suiza), el cual se ajustó para que el $90 \%$ de la malta molida pasara la criba US No. 30 (0.595 mm); lo cual equivale a la molienda fina de malta conforme el método oficial Malt-4 de la ASBC (ASBC, 2009). Para las pruebas de fermentación, donde se evaluó mayor cantidad de mosto y por tanto de malta molida, la molienda se llevó a cabo utilizando un molino de tres rodillos con tolva y motor marca nacional. La apertura entre los rodillos principales de este molino se ajustó para garantizar una retención sobre la criba US No. 30 de $75 \pm 1$ $\%$; equivalente a la molienda gruesa de malta. Debido a la diferencia en tamaños de partícula, el filtrado en la fase 1 se llevó a cabo con papel Ahlstrom No. 509 (Ahlstrom-Munksjö, Helsinki, Finlandia); mientras que el filtrado en la fase 2 en un recipiente cilíndrico de $5 \mathrm{~L}$ (con $25 \mathrm{~cm}$ de diámetro) provisto de salida circular en el fondo de $1 \mathrm{~cm}$ de diámetro que permitió la formación adecuada de la torta de filtrado facilitando el clarificado de mosto. La maceración de los tratamientos en ambas fases se llevó a cabo en un macerador de laboratorio Micromat $^{\mathrm{TM}}$ (Micromat Co., Mahwah, NJ, USA).

\section{Fase 1 \\ Experimento 1: Temperaturas de maceración}

Tratamiento 1 . Se pesaron $50 \mathrm{~g}$ de malta molida y se mezclaron con $200 \mathrm{~mL}$ de agua destilada a $67{ }^{\circ} \mathrm{C}$ en vasos de níquel de $500 \mathrm{~mL}$. Inmediatamente se colocaron en el macerador, el cual se mantuvo a $65^{\circ} \mathrm{C}$ con agitación constante (100 rpm) durante $5 \mathrm{~h}$. Sin embargo, dos vasos fueron retirados después de $1 \mathrm{~h}$, dos a las $2 \mathrm{~h}$, dos a las $4 \mathrm{~h}$ y tres a las $5 \mathrm{~h}$. Los vasos se enfriaron a $20^{\circ} \mathrm{C}$ y el contenido de cada uno se ajustó a 450 g con agua destilada a $20^{\circ} \mathrm{C}$ previo al filtrado. Este ajuste de peso se llevó a cabo en todos los tratamientos de la fase 1.

Tratamiento 2. Las condiciones de maceración y muestreo en este tratamiento fueron similares a las del tratamiento $1 ;$ con la diferencia de que la maceración se llevó a cabo a $60^{\circ} \mathrm{C}$.

Tratamiento 3. Las cantidades de malta molida y agua en este tratamiento fueron similares al tratamiento 1 , pero con diferentes temperaturas de maceración en un tiempo total de $5 \mathrm{~h}$ $\left(50^{\circ} \mathrm{C}, 1 \mathrm{~h} ; 55^{\circ} \mathrm{C}, 1 \mathrm{~h} ; 60^{\circ} \mathrm{C}, 1 \mathrm{~h} ; 65^{\circ} \mathrm{C}, 2 \mathrm{~h}\right)$. En este tratamiento no se hicieron muestreos intermedios.

\section{Experimento 2: Tiempo de maceración}

El mejor tratamiento en el experimento 1 fue la maceración isotérmica a $65{ }^{\circ} \mathrm{C}$. En consecuencia, se buscó determinar el mejor tiempo a dicha temperatura. Se utilizó la misma relación malta:agua de 1:4 (50 g malta $+200 \mathrm{~mL}$ agua); sin embargo, en este caso los tratamientos fueron $1 \mathrm{~h}$, $2 \mathrm{~h}, 3 \mathrm{~h}$ y $4 \mathrm{~h}$ de maceración a $65^{\circ} \mathrm{C}$.

\section{Experimento 3: Relación malta-agua}

El mejor tratamiento en el experimento 2 fue la maceración isotérmica a $65{ }^{\circ} \mathrm{C}$ por $3 \mathrm{~h}$. Debido a que la relación malta:agua utilizada en los experimentos 1 y 2 fue 1:4, que es la comúnmente utilizada en la elaboración de whisky, en este experimento se evaluaron las relaciones malta:agua 1:3 (50 g malta $+150 \mathrm{~mL}$ agua) y 1:5 (50 g malta $+250 \mathrm{~mL}$ agua); incluyendo la 1:4 nuevamente como testigo. 
Fase 2

\section{Experimento 4: Concentración de sólidos en mosto du-} rante la fermentación

En este experimento se evaluaron las condiciones de fermentación a diferentes niveles de sólidos solubles (grados Plato, ${ }^{\circ} \mathrm{P}$ ) iniciales en mosto. Los mostos se obtuvieron de una maceración a $65^{\circ} \mathrm{C}$ por $3 \mathrm{~h}$ en una relación malta:agua de 1:3. En este caso, tal como se observaría en un proceso comercial, el filtrado se llevó a cabo a través de la misma masa espesa que actúa como barrera donde las cascarillas de la malta facilitaron esta operación; misma que duró aproximadamente 40 min para $1.6 \mathrm{~kg}$ de malta en maceración. Este tiempo incluyó el retorno (recirculación) del mosto turbio (ca. 1.5 L) al recipiente de filtración una vez que la cama de filtrado generó mosto clarificado. Los tratamientos evaluados fueron $10{ }^{\circ} \mathrm{P}, 11.25^{\circ} \mathrm{P}, 12.5^{\circ} \mathrm{P}, 13.75^{\circ} \mathrm{P}, 15^{\circ} \mathrm{P}, 16.25^{\circ} \mathrm{P}$, y $17.5^{\circ} \mathrm{P}$. Para lograr estos niveles de sólidos solubles, en todos los casos se generaron mostos altamente concentrados $\left(20 \pm 0.5^{\circ} \mathrm{P}\right)$ y se diluyeron con un volumen calculado de agua destilada para el ajuste de sólidos en solución en función de los valores de gravedad específica, densidad del mosto a $20^{\circ} \mathrm{C}$ y el peso total de los sólidos en el mosto concentrado. De cada tratamiento se fermentaron $3 \mathrm{~L}$ de mosto con la levadura Fermentis SafAle ${ }^{\mathrm{TM}}$ S-04 (Grupo Lesaffre, Marcq-en-Baroeul, Francia). El inóculo se preparó con la adición de $1.725 \mathrm{~g}$ de levadura liofilizada a $300 \mathrm{~mL}$ de mosto tibio $\left(35^{\circ} \mathrm{C}\right)$; el cual se dejó reposar por 10 minutos para adecuada rehidratación. Posteriormente, el mosto inoculado se agitó vigorosamente y se mezcló con el resto del mosto a fermentar $(2.7 \mathrm{~L})$ en contenedores de vidrio ámbar de $4 \mathrm{~L}$ provistos de una trampa de oxígeno o 'air lock'. El tiempo de fermentación fue de 7 días (168 h) a $20{ }^{\circ} \mathrm{C}$. Para el análisis de variables respuesta, el mosto fermentado se filtró al vacío con papel Whatman GF/B (tamaño de poro de $1 \mu \mathrm{m}$ ) y de esta forma eliminar la levadura suspendida.

\section{Experimento 5: Temperatura y condiciones de fermenta- ción}

Se evaluaron tres diferentes temperaturas de fermentación $\left(20,25\right.$ y $\left.30{ }^{\circ} \mathrm{C}\right)$ en mostos con la mejor concentración inicial de sólidos observada en el experimento 4 (i.e. $12.5^{\circ} \mathrm{P}$ ). Las maceraciones se llevaron a cabo a $65^{\circ} \mathrm{C}$ por $3 \mathrm{~h}$ en una relación malta:agua de 1:3. La preparación del inóculo, tiempo de fermentación, y filtrado se llevó a cabo de forma similar a lo descrito en el experimento 4. Además, en este experimento se evaluaron condiciones de fermentación abierta (i.e. fermentación sin air lock) y cerrada (i.e. fermentación con air lock) a las temperaturas mencionadas.

\section{Variables respuesta y análisis estadístico}

En los experimentos 1-4 se utilizaron diseños experimentales completamente al azar; mientras que en el experimento 5 se usó un arreglo factorial para evaluar el efecto combinado de temperatura (factor A) y condiciones de fermentación (factor B) sobre las variables respuesta. Se hicieron tres repeticiones por tratamiento y la prueba de medias utilizada fue Tukey $(a=0.05)$. En el análisis de datos se utilizó el programa SAS versión 9.3 (SAS, 2011). Las vari-

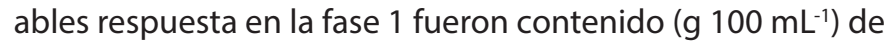
maltosa, glucosa, fructosa y maltotriosa en mosto; además de la suma de éstos como carbohidratos fermentables totales (CF) y la fracción más fermentable (CF+) que excluye a la maltotriosa, carbohidrato parcialmente consumido durante la fermentación (Stewart, 2009). Debido a que todos los macerados en la fase 1 se ajustaron al mismo peso (450 g) previo al filtrado, no se observó variación significativa en el contenido de sólidos ( $\left.8.9^{\circ} \mathrm{P}\right)$ de los mostos resultantes; por lo que este parámetro no se consideró como una variable respuesta. Asimismo, el FAN en mosto no fue un factor limitante para el desarrollo de la levadura debido a que las 200 ppm observadas garantizan una fermentación eficiente (Hill y Stewart, 2019). En la fase 2, las variables respuesta fueron contenido de etanol $(\% \mathrm{v} / \mathrm{v})$, rendimiento de etanol (g etanol/g sólidos solubles), metanol (\% v/v) y glicerol (g $\left.\mathrm{L}^{-1}\right)$; además del contenido de CF residuales, con los que se calculó la fermentabilidad de CF en función de la reducción porcentual de CF inicial - CF residual. Para el cálculo de la variable rendimiento de etanol se tomó como volumen base $100 \mathrm{~mL}$, donde se dividió el etanol (g) presente en dicho volumen de mosto fermentado entre la cantidad de sólidos (g) presentes en el mismo volumen de mosto sin fermentar.

El análisis de CF en mosto, así como los derivados de fermentación (etanol, metanol y glicerol), se llevó a cabo por HPLC en un cromatógrafo marca Agilent Technologies 1100, provisto de un detector de índice de refacción y una columna Aminex ${ }^{\circledR}$ HPX-87H de 300 x 7.8 mm marca Bio-Rad. Se inyectaron $20 \mu \mathrm{L}$ de muestra filtrada $(0.45 \mu \mathrm{m})$ en una fase móvil de ácido sulfúrico $0.008 \mathrm{M}$ a un flujo de $0.6 \mathrm{~mL} \mathrm{~min}{ }^{-1}$, con temperatura de la columna de $65^{\circ} \mathrm{C}$. Los valores de CF se determinaron utilizando una mezcla estándar en $\mathrm{g} 100 \mathrm{~mL}^{-1}$ como lo indica el método Wort-14 de los métodos oficiales de la ASBC (ASBC, 2009). El análisis de etanol (\% v/v), metanol (\% v/v) y glicerol $\left(\mathrm{g} \mathrm{L}^{-1}\right.$ ) se llevó a cabo utilizando los cromatogramas con las áreas respuesta de estos compuestos diluidos a diferentes concentraciones en agua destilada (muestras estándar). Las áreas respuesta de cada muestra estándar se graficaron para obtener curvas de regresión lineal; mismas que se utilizaron para calcular su concentración en mosto fermentado a partir del área respuesta observada en el mismo tiempo de retención que la muestra estándar.

\section{RESULTADOS Y DISCUSIÓN}

Fase 1

Los resultados del experimento 1 (Tabla 1) mostraron que después de $5 \mathrm{~h}$ de maceración isotérmica a $60{ }^{\circ} \mathrm{C}$ o 65 ${ }^{\circ} \mathrm{C}$ se genera mayor concentración de CF en mosto en comparación con la maceración de temperaturas escalonadas (50-55-60-65 $\left.{ }^{\circ} \mathrm{C}\right)$. Asimismo, los muestreos intermedios en las maceraciones isotérmicas mostraron que después de $2 \mathrm{~h}$ de maceración se cuenta con el $90 \%$ de CF determinados en 5 h; mientras que con $4 \mathrm{~h}$ de maceración el valor de CF fue mayor a $99 \%$ en ambos tratamientos isotérmicos. Similar a 


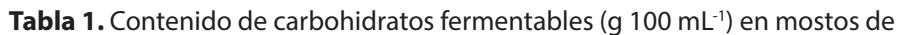
tres tratamientos de maceración a diferentes temperaturas (experimento 1). Table 1. Fermentable carbohydrates content ( $100 \mathrm{~mL}^{-1}$ ) in worts, from three mashing treatments at different temperatures (experiment 1).

\begin{tabular}{ccccccc}
\hline Tratamiento* & Glucosa & Fructosa & Maltosa & Maltotriosa & CF** & CF+*** \\
\hline 1 & $1.25 \mathrm{a}$ & $0.26 \mathrm{a}$ & $5.24 \mathrm{a}$ & $1.08 \mathrm{~b}$ & $7.84 \mathrm{a}$ & $6.76 \mathrm{a}$ \\
2 & $1.05 \mathrm{a}$ & $0.21 \mathrm{a}$ & $5.26 \mathrm{a}$ & $1.17 \mathrm{ab}$ & $7.69 \mathrm{ab}$ & $6.52 \mathrm{a}$ \\
3 & $1.40 \mathrm{a}$ & $0.23 \mathrm{a}$ & $4.60 \mathrm{~b}$ & $1.18 \mathrm{a}$ & $7.41 \mathrm{~b}$ & $6.23 \mathrm{~b}$ \\
\hline
\end{tabular}

${ }^{*} 1: 65^{\circ} \mathrm{C}, 5 \mathrm{~h} ; 2: 60^{\circ} \mathrm{C}, 5 \mathrm{~h} ; 3: 50{ }^{\circ} \mathrm{C}, 1 \mathrm{~h} ; 55^{\circ} \mathrm{C}, 1 \mathrm{~h} ; 60^{\circ} \mathrm{C}, 1 \mathrm{~h} ; 65^{\circ} \mathrm{C}, 2 \mathrm{~h}{ }^{* *} \mathrm{C} . \mathrm{F} .=-$ Carbohidratos fermentables totales. ${ }^{* * *}$ Carbohidratos más fermentables.

Medias con diferente letra entre tratamientos implican diferencia significativa (Tukey, $a=0.05$ ).

estos resultados, Evans et al. (2005) reportaron que las temperaturas iniciales de maceración, mantenidas por al menos 50 min, afectan la composición del mosto en términos de CF. Ellos evaluaron temperaturas iniciales en el rango $45-76^{\circ} \mathrm{C}$ y observaron que la temperatura de $65^{\circ} \mathrm{C}$ produce mostos con mayor contenido de CF; mientras que a temperaturas superiores se observa una rápida reducción de maltosa a expensas del incremento de carbohidratos no fermentables como maltotetraosa. El incremento de este carbohidrato se debe principalmente a que, mientras la beta amilasa pierde actividad a temperaturas altas $\left(>65^{\circ} \mathrm{C}\right)$ dejando de generar maltosa, la alfa amilasa se mantiene estable; generando múltiples oligosacáridos, incluyendo la maltotetraosa (Muralikrishna y Nirmala, 2005; Henson et al., 2014). En este estudio, se observó un incremento significativo en el contenido de maltosa cuando se maceró a 60 y $65^{\circ} \mathrm{C}$ en comparación con la maceración de temperaturas escalonadas; cuyos valores promedio fueron

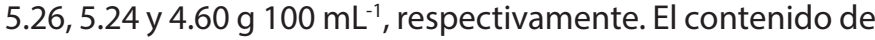
maltosa observado en los mostos de las maceraciones isotérmicas por $5 \mathrm{~h}$ es significativamente mayor a otros reportados a similar temperatura de maceración con fines cerveceros. Por ejemplo, Evans et al. (2005) reportaron un contenido de

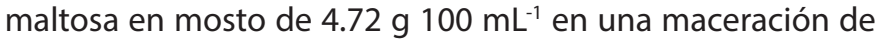
$50 \mathrm{~min}$ a $65^{\circ} \mathrm{C}$ con posterior incremento a $70^{\circ} \mathrm{C}$ mantenidos por 30 min; mientras que Schwarz et al. (2007) reportaron un

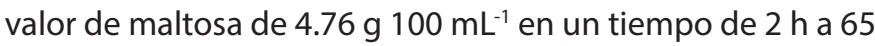
${ }^{\circ} \mathrm{C}$ con incremento posterior a $75^{\circ} \mathrm{C}$ mantenidos por $10 \mathrm{~min}$. Respecto al contenido total de CF en mosto, ambos autores

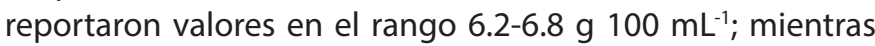
que los valores de este parámetro en el experimento 1 es-

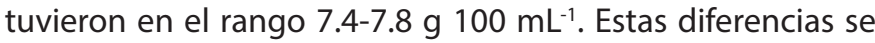
deben a que en cervecería la degradación enzimática de la amilopectina es parcial, por lo que en un mosto cervecero es común observar que la maltosa constituye el $50-60 \%$ del total de carbohidratos; mientras que las dextrinas el 20-30 $\%$ (Stewart, 2009). Por el contrario, en mostos para whisky se busca la mayor degradación posible del almidón; por lo que la presencia de dextrinas debe reducirse al mínimo con el objetivo de lograr el mayor rendimiento de etanol por unidad de malta (Lyons, 2003). En este estudio, se estima que la presencia de carbohidratos no fermentables (dextrinas + oligosacáridos) en los mostos evaluados con $8.9^{\circ} \mathrm{P}$ fue menor a $15 \%$ en la maceración escalonada, y menor a $12 \%$ y 10 $\%$ en las maceraciones isotérmicas a 60 y $65^{\circ} \mathrm{C}$, respectiva- mente. Esta estimación se llevó a cabo asumiendo que 90-92 $\%$ de los sólidos en mosto son carbohidratos (Willaert, 2007) y que el valor de proteína soluble de la malta utilizada (5.3 $\%$ b.s.) equivale, considerando $85 \%$ de extracto de malta, a $0.56^{\circ} \mathrm{P}\left(6.3 \%\right.$ de $\left.8.9^{\circ} \mathrm{P}\right)$; además de que la concentración reportada del tercer componente menor en mosto (i.e. lípidos) generalmente equivale $\mathrm{a}<0.002^{\circ} \mathrm{P}\left(<20 \mathrm{mg} \mathrm{L}^{-1}\right)$ de los sólidos cuando se macera a $65^{\circ} \mathrm{C}$ (Evans et al., 2012). Considerando lo anterior, además de la relación porcentual $\mathrm{CF}+/ \mathrm{CF}$ que fue de $86 \%, 85 \%$ y $84 \%$ para los tratamientos 1,2 y 3 , respectivamente, se determinó que la mejor condición de maceración en el experimento 1 es isotérmica a $65^{\circ} \mathrm{C}$. El incremento de la relación porcentual $\mathrm{CF}+/ \mathrm{CF}$ implica mayor fermentabilidad del total de carbohidratos fermentables en mosto en función de la reducción del carbohidrato parcialmente fermentable, maltotriosa. El hecho de que los muestreos intermedios bajo las condiciones isotérmicas del experimento 1 hayan mostrado que después de $4 \mathrm{~h}$ de maceración ya no existen incrementos significativos de CF, justificó este tiempo como el máximo a evaluar en el experimento 2 . Se observó que no existe diferencia en la generación de CF cuando se macera por tres o cuatro horas a $65^{\circ} \mathrm{C}$ (Tabla 2). A pesar de que las temperaturas de maceración utilizadas en la producción de whisky de malta oscilan en el rango $55-65{ }^{\circ} \mathrm{C}$; los tiempos de maceración (sin contabilizar el tiempo de filtrado de mosto) generalmente varían entre 1 y $2.5 \mathrm{~h}$. El parámetro que influye en la variabilidad de estos tiempos es DP, cuyas enzimas constituyentes (alfa amilasa, beta amilasa, limite dextrinasa, y alfa glucosidasa; i.e. enzimas diastásicas) son las responsables de desdoblar el almidón a CF (Evans et al., 2010). En esta investigación, se utilizó una malta con poder diastático intermedio; sin embargo, es posible que con un DP mayor ( $>150^{\circ} \mathrm{ASBC}$ ), el tiempo de maceración para lograr el máximo contenido de CF podría haber presentado una ligera reducción. No obstante, el uso maltas con alto DP en la producción de whisky de malta tiene el inconveniente de que existe una correlación positiva entre DP y PT; así como negativa entre PT y extracto de malta (Huerta et al., 2014). Esto se debe a que la enzima que mayor contribuye al DP, beta amilasa (Gibson et al., 1995), está ligada al contenido total de proteína en cebada debido a que la síntesis y acumulación de esta enzima se da en la etapa de llenado de grano durante el desarrollo del cultivo de cebada; lo cual explica su alta correlación con las hordeínas (Qi et al., 2006; Wei et al., 
Tabla 2. Contenido de carbohidratos fermentables ( $100 \mathrm{~mL}^{-1}$ ) en mostos de diferentes tiempos de maceración isotérmica a $65^{\circ} \mathrm{C}$ (experimento 2).

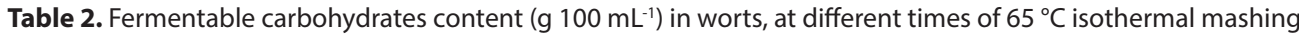
(experiment 2).

\begin{tabular}{ccccccc}
\hline Tratamiento* & Glucosa & Fructosa & Maltosa & Maltotriosa & CF** & CF+*** \\
\hline 1 & $0.98 \mathrm{a}$ & $0.17 \mathrm{~b}$ & $5.26 \mathrm{a}$ & $1.23 \mathrm{a}$ & $7.64 \mathrm{a}$ & $6.40 \mathrm{a}$ \\
2 & $1.01 \mathrm{a}$ & $0.17 \mathrm{ab}$ & $5.33 \mathrm{a}$ & $1.27 \mathrm{a}$ & $7.77 \mathrm{a}$ & $6.51 \mathrm{a}$ \\
3 & $1.04 \mathrm{a}$ & $0.18 \mathrm{ab}$ & $5.50 \mathrm{~b}$ & $1.23 \mathrm{a}$ & $7.95 \mathrm{~b}$ & $6.72 \mathrm{~b}$ \\
4 & $1.04 \mathrm{a}$ & $0.19 \mathrm{a}$ & $5.48 \mathrm{ab}$ & $1.25 \mathrm{a}$ & $7.96 \mathrm{ab}$ & $6.71 \mathrm{ab}$ \\
\hline
\end{tabular}

${ }^{*} 1: 65^{\circ} \mathrm{C}, 1 \mathrm{~h} ; 2: 65^{\circ} \mathrm{C}, 2 \mathrm{~h} ; 3: 65^{\circ} \mathrm{C}, 3 \mathrm{~h} ; 4: 65^{\circ} \mathrm{C}, 4$ h. ${ }^{* *} \mathrm{C} . \mathrm{F} .=$ Carbohidratos fermentables totales. ${ }^{* *} \mathrm{Carbohidratos}$ más fermentables. Medias con diferente letra entre tratamientos implican diferencia significativa (Tukey, $\mathrm{a}=0.05)$.

2009). En consecuencia, a mayor PT, mayor DP; pero menor extracto de malta. En la elaboración de whiskies de grano, donde se utilizan granos sin maltear más una proporción de malta de cebada, es deseable que las maltas utilizadas posean alto DP; sin embargo, en whisky de malta se observaría una reducción no deseable de rendimiento de etanol debido a la reducción en extracto de malta. Es por esta razón que las especificaciones de DP para maltas destinadas a whisky de malta indican valores menores a $150^{\circ} \mathrm{ASBC}$; mientras que para las maltas destinadas a whisky de grano el DP puede ir más allá de $200{ }^{\circ} \mathrm{ASBC}$ (Bringhurst y Brosnan, 2014; AMBA, 2021). Por lo tanto, es poco probable que el tiempo óptimo de maceración de $3 \mathrm{~h}$ determinado en esta investigación para la producción de whisky de malta se reduzca de forma considerable debido al efecto negativo de usar maltas con alto DP y reducido extracto de malta en el rendimiento de etanol.

Por otro lado, con los resultados del experimento 3 se observó que la condición isotérmica a $65^{\circ} \mathrm{C}$ por 3 h y relación malta:agua 1:3 genera mayor contenido de maltosa y $\mathrm{CF}+$ (Tabla 3). A pesar del mayor valor promedio de CF en los mostos de la relación 1:3, no se observó diferencia estadística de este parámetro entre los tres tratamientos del experimento 3 , lo cual pudo deberse a la mayor dispersión de valores de CF que mostraron los mostos 1:4 y 1:5. Sin embargo, esto no ocurrió con $\mathrm{CF}+$, donde sí se observa diferencia significativa y favorable a la relación 1:3; misma que presentó la mayor relación porcentual CF+/CF (85.4\%). En estudios previos, los altos valores de $\mathrm{CF}$ en mostos con menor relación malta:agua han sido vinculados a la estabilidad térmica de las enzimas diastásicas; cuya tolerancia a altas temperaturas es mayor en soluciones con cierto nivel de sólidos que cuando éstas se encuentran aisladas (Muller, 1991; Stenholm y Home, 1999; Evans et al., 2011). Si bien el contenido de CF se reduce significativamente a temperaturas altas $\left(>70^{\circ} \mathrm{C}\right)$, el efecto es menor a medida que incrementa la concentración de sólidos durante la maceración. Evans y Fox (2017) reportaron que bajo las condiciones de maceración del Mosto Congreso, con temperaturas de maceración en el rango $45-70{ }^{\circ} \mathrm{C}$ y relación malta:agua 1:4 (ASBC, 2009), la actividad de beta amilasa en la malta de diferentes variedades de cebada se reduce drásticamente (de $85 \%$ a $<10 \%$ ) cuando el macerado transcurre en el rango de temperatura $65-70{ }^{\circ} \mathrm{C}$; mientras que en la maceración isotérmica a $65^{\circ} \mathrm{C}$, con relación malta:agua 1:3, la beta amilasa retiene $40-70 \%$ de su actividad después de 60 min. A pesar de que Evans y Fox (2017) no reportaron el perfil de carbohidratos en los mostos obtenidos, sus observaciones respecto al comportamiento de beta amilasa permiten entender la razón del alto contenido de maltosa y CF+ en mostos de maceraciones isotérmicas a $65^{\circ} \mathrm{C}$; asumiendo que esta enzima mantiene cierta actividad residual después de $3 \mathrm{~h}$ de incubación.

\section{Fase 2}

Con la maceración isotérmica a $65^{\circ} \mathrm{C}$ por $3 \mathrm{~h}$ y relación malta:agua 1:3 se obtuvieron los mostos para los experimentos de fermentación. Debido a que el ajuste de sólidos previo a la fermentación de los diferentes tratamientos se hizo a partir de mostos altamente concentrados $\left(20 \pm 0.5^{\circ} \mathrm{P}\right)$, se observó un incremento proporcional en cuanto a carbohidratos fermentables en los tratamientos del experimento 4 (Tabla 4). Sin embargo, como se esperaba, la relación porcentual $\mathrm{CF}+/ \mathrm{CF}$ a diferentes niveles de sólidos en mosto se mantuvo sin mayor variación (83.3 $\pm 0.3 \%$ ); así como el contenido porcentual de maltosa respecto a CF, que osciló en el rango 66$68 \%$. El valor de $\mathrm{CF}+/ \mathrm{CF}$ en este experimento es ligeramente menor al observado (85.4\%) bajo las mismas condiciones de maceración de la fase 1; no obstante, esta diferencia se atribuye al molido grueso llevado a cabo en esta etapa con el fin de emular condiciones comerciales. Al incrementar el

Tabla 3. Contenido de carbohidratos fermentables $\left(\mathrm{g} 100 \mathrm{~mL}^{-1}\right)$ en mostos de maceraciones isotérmicas $\left(65^{\circ} \mathrm{C}, 3 \mathrm{~h}\right)$ y diferentes relaciones malta:agua (experimento 3 ).

Table 3. Fermentable carbohydrates content $\left(\mathrm{g} 100 \mathrm{~mL}^{-1}\right)$ in worts from isothermal mashings $\left(65^{\circ} \mathrm{C}, 3 \mathrm{~h}\right)$, and different grist:water ratios (experiment 3).

\begin{tabular}{ccccccc}
\hline Tratamiento* & Glucosa & Fructosa & Maltosa & Maltotriosa & CF** & CF+*** \\
\hline 1 & $1.09 \mathrm{a}$ & $0.19 \mathrm{a}$ & $5.16 \mathrm{a}$ & $1.10 \mathrm{~b}$ & $7.55 \mathrm{a}$ & $6.45 \mathrm{a}$ \\
2 & $0.99 \mathrm{~b}$ & $0.18 \mathrm{a}$ & $4.91 \mathrm{~b}$ & $1.16 \mathrm{~b}$ & $7.25 \mathrm{a}$ & $6.09 \mathrm{~b}$ \\
3 & $0.96 \mathrm{~b}$ & $0.18 \mathrm{a}$ & $5.11 \mathrm{ab}$ & $1.26 \mathrm{a}$ & $7.47 \mathrm{a}$ & $6.22 \mathrm{ab}$ \\
\hline
\end{tabular}

${ }^{*} 1=1: 3 ; 2=1: 4 ; 3=1: 5$. ${ }^{*}$ C.F.=Carbohidratos fermentables totales. ${ }^{* *}$ Carbohidratos más fermentables. Medias con diferente letra entre tratamientos implican diferencia significativa (Tukey, $\mathrm{a}=0.05$ ). 
Tabla 4. Contenido de carbohidratos fermentables $\left(\mathrm{g} 100 \mathrm{~mL}^{-1}\right)$ en mostos a diferentes niveles de sólidos solubles $\left({ }^{\circ} \mathrm{P}\right)$ generados de un mosto concentrado $\left(20^{\circ} \mathrm{P}\right)$ obtenido de una maceración isotérmica a $65^{\circ} \mathrm{C}$ por $3 \mathrm{~h}$ y relación malta:agua $1: 3$ (experimento 4).

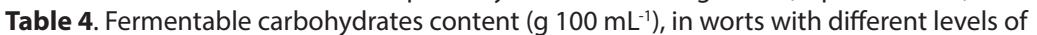
soluble solids ( $\left.{ }^{\circ} \mathrm{P}\right)$ adjusted from a concentrated wort $\left(20^{\circ} \mathrm{P}\right)$, obtained with an isothermal mashing at $65^{\circ} \mathrm{C}$ for $3 \mathrm{~h}$ and a 1:3 grist:water ratio (experiment 4).

\begin{tabular}{ccccccc}
\hline Tratamiento* & Glucosa & Fructosa & Maltosa & Maltotriosa & CF** & CF+*** \\
\hline 10.00 & $1.15 \mathrm{a}$ & $0.21 \mathrm{~b}$ & $5.91 \mathrm{~d}$ & $1.48 \mathrm{~d}$ & $8.75 \mathrm{c}$ & $7.28 \mathrm{c}$ \\
11.25 & $1.49 \mathrm{~b}$ & $0.32 \mathrm{ab}$ & $6.70 \mathrm{~cd}$ & $1.65 \mathrm{~cd}$ & $10.17 \mathrm{bc}$ & $8.52 \mathrm{bc}$ \\
12.50 & $1.35 \mathrm{~b}$ & $0.25 \mathrm{ab}$ & $7.01 \mathrm{bcd}$ & $1.76 \mathrm{bcd}$ & $10.36 \mathrm{bc}$ & $8.61 \mathrm{bc}$ \\
13.75 & $1.60 \mathrm{bc}$ & $0.37 \mathrm{ab}$ & $7.70 \mathrm{bcd}$ & $1.92 \mathrm{abcd}$ & $11.59 \mathrm{abc}$ & $9.67 \mathrm{abc}$ \\
15.00 & $1.64 \mathrm{c}$ & $0.31 \mathrm{ab}$ & $8.37 \mathrm{abc}$ & $2.13 \mathrm{abc}$ & $12.44 \mathrm{ab}$ & $10.31 \mathrm{ab}$ \\
16.25 & $1.90 \mathrm{~cd}$ & $0.39 \mathrm{a}$ & $8.77 \mathrm{ab}$ & $2.20 \mathrm{ab}$ & $13.26 \mathrm{ab}$ & $11.06 \mathrm{ab}$ \\
17.50 & $1.91 \mathrm{~d}$ & $0.34 \mathrm{ab}$ & $9.70 \mathrm{a}$ & $2.39 \mathrm{a}$ & $14.34 \mathrm{a}$ & $11.95 \mathrm{a}$ \\
\hline
\end{tabular}

*Sólidos solubles en ${ }^{\circ} \mathrm{P}{ }^{* *} \mathrm{C} . \mathrm{F} .=$ Carbohidratos fermentables totales. ${ }^{* *}$ Carbohidratos más fermentables. Medias con diferente letra entre tratamientos implican diferencia significativa (Tukey, $a=0.05$ ).

tamaño de partícula durante la molienda de malta, además de la reducción del contenido de sólidos solubles en mosto, disminuye la fermentabilidad de mostos y contenido de alcohol en mosto fermentado (Evans et al., 2011); parámetros altamente asociados a CF y CF+/CF (Cutaia, 2007).

Como consecuencia del incremento en los sólidos solubles, los mostos fermentados del experimento 4 mostraron aumento gradual del contenido de etanol (Tabla 5). No obstante, al considerar el parámetro 'rendimiento de etanol', se observó que los mejores tratamientos fueron $12.5^{\circ} \mathrm{P}$ y 15.0 ${ }^{\circ} \mathrm{P}$, superiores al resto de los tratamientos en promedio 0.02 $\mathrm{g}$ etanol/g sólidos solubles; lo cual, haciendo los cálculos respectivos, equivale a $\sim 20 \mathrm{~L}$ de etanol puro por tonelada de malta. Por otro lado, la menor generación de metanol ocurrió con los tratamientos $10.0^{\circ} \mathrm{P}$ y $12.5^{\circ} \mathrm{P}$; mismos que mostraron reducidos niveles de glicerol. La presencia de metanol es una característica indeseable en bebidas alcohólicas debido a su toxicidad, por lo que diferentes países establecen límites máximos respecto a este compuesto tanto en bebidas fermentadas como destiladas (Ohimain, 2016). A pesar de que la mayor parte del metanol se elimina en las etapas iniciales de la destilación como parte de los foreshots o 'cabezas', bajas concentraciones de este compuesto contribuyen a la mejor recuperación de etanol en las fracciones intermedias del destilado (Lyons, 2003; Nicol, 2014; Kwak et al., 2015).
Respecto al glicerol, se observó incremento significativo en mostos fermentados a medida que la cantidad inicial de sólidos solubles fue mayor. El incremento de glicerol durante la fermentación de soluciones con mayor presión osmótica (i.e. $\left.>^{\circ} \mathrm{P}\right)$, es una respuesta de la levadura que genera y acumula este compuesto para incrementar su tolerancia a dichas condiciones de estrés (Nevoigt y Stahl, 1997; Cronwright et al., 2002). A pesar de que el glicerol es deseable hasta cierto punto en bebidas fermentadas como la cerveza y el vino dado su efecto positivo en palatabilidad y percepción de sabor, en la producción de whisky constituye una desventaja por dos razones principales: 1) su punto de ebullición es de $290^{\circ} \mathrm{C}$, por lo que no es un compuesto presente en esta bebida (Aylott, 2014); y 2) su biosíntesis (al igual que la de etanol) se lleva a cabo a partir de glucosa, resultando una relación inversa entre ambos compuestos (Scanes et al., 1998; Aslankoohi et al., 2015; Li et al., 2015). Además del glicerol, se ha reportado la variación de otros compuestos (tanto deseables como no deseables) generados en fermentaciones con alto contenido de sólidos solubles (Younis y Stewart, 1999); comúnmente denominadas fermentaciones de alta gravedad, por implicar alta gravedad específica de los mostos a fermentar (i.e. gravedad específica a $20^{\circ} \mathrm{C} \geq 1.061$, equivalente $\mathrm{a} \geq 15^{\circ} \mathrm{P}$ ). El uso de fermentaciones de alta gravedad en cervecería, a pesar de inconvenientes observados en la reducción de la estabilidad

Tabla 5. Características de mostos de malta de cebada fermentados a $20^{\circ} \mathrm{C}(168 \mathrm{~h})$ con diferentes niveles de sólidos solubles ( $\left.{ }^{\circ} \mathrm{P}\right)$ iniciales.

Table 5. Characteristics of malt worts fermented at $20^{\circ} \mathrm{C}(168 \mathrm{~h})$ with different levels of initial soluble solids ( $\left.{ }^{\circ} \mathrm{P}\right)$.

\begin{tabular}{ccccccc}
\hline${ }^{\circ} \mathbf{P}$ & Etanol $(\% \mathbf{v} / \mathbf{v})$ & Etanol $_{\mathbf{R}}(\mathbf{g} / \mathbf{g})^{1}$ & $\mathbf{M e t a n o l}(\% \mathbf{v} / \mathbf{v})$ & $\mathbf{G}_{\mathrm{OH}}\left(\mathbf{g ~ L}^{-1}\right)^{\mathbf{2}}$ & $\mathbf{M 3}_{\mathrm{F}}(\%)^{3}$ & $\mathbf{C F}_{\mathrm{F}}(\%)^{4}$ \\
\hline 10.00 & $4.23 \mathrm{~d}$ & $0.32 \mathrm{~b}$ & $0.02 \mathrm{~b}$ & $2.02 \mathrm{~d}$ & $58.89 \mathrm{~b}$ & $90.05 \mathrm{a}$ \\
11.25 & $4.74 \mathrm{~d}$ & $0.32 \mathrm{~b}$ & $0.07 \mathrm{c}$ & $2.66 \mathrm{bc}$ & $62.95 \mathrm{ab}$ & $91.06 \mathrm{c}$ \\
12.50 & $5.71 \mathrm{c}$ & $0.34 \mathrm{c}$ & $0.02 \mathrm{~b}$ & $2.55 \mathrm{c}$ & $64.68 \mathrm{ab}$ & $91.94 \mathrm{bc}$ \\
13.75 & $6.14 \mathrm{bc}$ & $0.33 \mathrm{bc}$ & $0.07 \mathrm{c}$ & $3.06 \mathrm{ab}$ & $70.62 \mathrm{ab}$ & $93.03 \mathrm{~b}$ \\
15.00 & $6.89 \mathrm{ab}$ & $0.34 \mathrm{c}$ & $0.05 \mathrm{c}$ & $2.86 \mathrm{abc}$ & $70.33 \mathrm{ab}$ & $93.16 \mathrm{~b}$ \\
16.25 & $7.06 \mathrm{ab}$ & $0.32 \mathrm{~b}$ & $0.05 \mathrm{c}$ & $3.30 \mathrm{a}$ & $76.94 \mathrm{a}$ & $93.49 \mathrm{~b}$ \\
\hline 17.50 & $7.13 \mathrm{a}$ & $0.30 \mathrm{a}$ & $0.17 \mathrm{a}$ & $3.13 \mathrm{a}$ & $71.11 \mathrm{ab}$ & $92.92 \mathrm{~b}$ \\
\hline
\end{tabular}

${ }^{1}$ Rendimiento de etanol (g etanol/g sólidos solubles); ${ }^{2}$ Glicerol; ${ }^{3}$ Fermentabilidad de maltotriosa (\%, inicial-final); ${ }^{4}$ Fermentabilidad de carbohidratos fermentables totales (\%, inicial-final). Medias con diferente letra entre tratamientos implican diferencia significativa (Tukey, $\mathrm{a}=0.05$ ). 
de espuma asociada al incremento de la Proteinasa $A$, ha resultado benéfica respecto a maximizar el uso de espacio en plantas cerveceras $y$, en consecuencia, el incremento en la capacidad de producción. No obstante, en fermentaciones a $>20^{\circ} \mathrm{P}$ para whisky de grano, se ha observado que el patrón fermentativo de la levadura cambia considerablemente al incrementar el contenido de maltosa y maltotriosa residuales en mostos fermentados; lo que genera bajo contenido de etanol (Stewart, 2009; Stewart, 2010). En este estudio, a pesar de la reducción significativa del rendimiento de etanol a 17.5 ${ }^{\circ} \mathrm{P}$, no se observó variación respecto a la fermentabilidad de carbohidratos fermentables entre tratamientos; lo que sugiere que la levadura, previo a modificar su patrón de consumo de carbohidratos, reduciría inicialmente la síntesis de etanol. Considerando los resultados observados en el experimento 4 , se concluye que la mejor concentración de sólidos solubles para el incremento de etanol en fermentaciones de whisky de malta es $12.5^{\circ} \mathrm{P}$.

En el experimento 5 se utilizaron mostos de 12.5 ${ }^{\circ} \mathrm{P}$, por lo que no se observó diferencia estadística en cada uno de los mostos obtenidos por separado a este nivel de sólidos solubles respecto a $\mathrm{CF}$ y $\mathrm{CF}+$, cuyos valores promedio

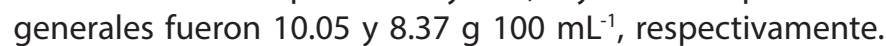
Asimismo, tanto los valores de maltosa como la relación $\mathrm{CF}+$ / CF fueron similares a lo observado en el experimento 4 con los mostos a $12.5^{\circ} \mathrm{P}$. Los resultados de este experimento señalan que, en términos de rendimiento de etanol, la mejor temperatura de fermentación de las tres evaluadas en el Factor $\mathrm{A}$, a través de los tratamientos del Factor $\mathrm{B}$, es $20^{\circ} \mathrm{C}$ (Tabla 6). Este resultado confirma la importancia del control de temperaturas en la producción de whisky, ya que -a diferencia de las fermentaciones cerveceras- las fermentaciones en destilería son generalmente menos estrictas (Russell y Stewart, 2014). Por otro lado, la fermentación cerrada, a través de los tratamientos del factor $\mathrm{A}$, mostró mayor rendimiento de etanol que la fermentación abierta; lo cual es atribuible a las características de fermentación de la levadura debido a que, a pesar de que este microorganismo optará por la ruta anaeróbica considerando el nivel de sólidos solubles presentes, la presencia de oxígeno contribuye al incremento del número de células en suspensión; lo cual ocurre a expensas de CF (Rosenfeld et al., 2003; Cutaia, 2007). Además, al analizar las condiciones de fermentación a $20^{\circ} \mathrm{C}$ se observó que en fermentación abierta se incrementa en $14 \%$ el glicerol y disminuye el rendimiento de etanol en $0.01 \mathrm{~g}$ etanol $/ \mathrm{g}$ sólidos solubles (equivalente a $\sim 10 \mathrm{~L}$ de etanol puro por tonelada de malta) respecto a la fermentación cerrada. A pesar de que actualmente en cervecería es difícil encontrar fermentaciones abiertas, en la producción de destilados como el whisky aún se utilizan fermentadores abiertos; aunque en décadas recientes su uso ha disminuido gradualmente para dar paso a los fermentadores de acero inoxidable. No obstante, este cambio más bien se ha dado con el objetivo de disminuir la contaminación bacteriana y de levaduras salvajes; cuyos metabolitos causan efectos negativos en el perfil de sabor de destilados (Wilson, 2014).

\section{CONCLUSIONES}

Las variaciones en las condiciones de maceración afectan significativamente el contenido de $\mathrm{CF}$ en mosto. Se determinó que la maceración a $65{ }^{\circ} \mathrm{C}$ durante $3 \mathrm{~h}$ con relación malta:agua 1:3 resulta en la generación de mostos con mayor contenido de CF, principalmente los tres más fermentables (i.e. fructosa, glucosa, maltosa); por lo que bajo estas condiciones mejoró sustancialmente la relación $\mathrm{CF}+/$ CF. El mejor rendimiento de etanol $(0.34 \mathrm{~g}$ etanol $/ \mathrm{g}$ sólidos solubles) durante la fermentación de estos mostos se obtuvo en fermentaciones cerradas a $12.5^{\circ} \mathrm{P}$ y $20^{\circ} \mathrm{C}$. Se observó que las fermentaciones de alta gravedad $\left(>15^{\circ} \mathrm{P}\right)$ resultan en el incremento de glicerol y reducción en el rendimiento de etanol.

\section{REFERENCIAS}

Agu, R.C., Bringhurst, T.A., and Brosnan, J.M. 2006. Production of grain whisky and ethanol from wheat, maize and other Cereals. Journal of the Institute of Brewing 112(4): 314-323.

American Malting Barley Association, Inc. (AMBA). Malting barley breeding guidelines, ideal commercial malt criteria. [Consultado el 4 de septiembre de 2021] 2021. Disponible en: https://ambainc.org/wp-content/uploads/2021/07/ Malting-Barley-Breeding-Guidelines_2021_Jun e.pdf.

American Society of Brewing Chemists (ASBC). 2009. Methods of Analysis, 8th Ed. Malt-3 Moisture, -4 Extract, -6 A Diastatic power, -7A Alpha-amylase, -8A Protein (Nx6.25) by Kjeldahl, -12 Malt modification by friability; Wort-2B Specific gravity

Tabla 6. Características de mostos de malta de cebada fermentados a $12.5^{\circ} \mathrm{P}$, tres diferentes temperaturas (Factor A) y dos condiciones de fermentación (Factor B).

Table 6. Characteristics of malt worts fermented at $12.5^{\circ} \mathrm{P}$, three different temperatures (Factor A), and two fermentation conditions (Factor B).

\begin{tabular}{|c|c|c|c|c|c|c|c|}
\hline Factor ${ }^{1}$ & Nivel $^{2}$ & Etanol (\%v/v) & Etanol $_{R}(g / g)^{3}$ & Metanol (\%v/v) & $\mathbf{G}_{\mathrm{OH}}\left(\mathrm{g} \mathrm{L}^{-1}\right)^{4}$ & M3 $3_{F}(\%)^{5}$ & $\mathrm{CF}_{\mathrm{F}}(\%)^{6}$ \\
\hline & 1 & $5.58 \mathrm{a}$ & $0.34 a$ & $0.04 a$ & 2.73ab & $63.33 b$ & $91.78 b$ \\
\hline \multirow[t]{2}{*}{ A } & 2 & $4.41 \mathrm{~b}$ & $0.27 b$ & $0.07 a$ & $2.52 a$ & $81.39 a$ & $95.28 a$ \\
\hline & 3 & $4.75 a b$ & $0.29 a b$ & $0.11 a$ & $3.02 b$ & $68.84 a b$ & $93.37 a b$ \\
\hline \multirow[t]{2}{*}{ B } & 1 & $4.55 a$ & $0.27 a$ & $0.08 a$ & $2.79 a$ & $70.16 a$ & $93.22 a$ \\
\hline & 2 & $5.27 \mathrm{~b}$ & $0.32 b$ & $0.07 a$ & $2.72 a$ & $72.21 \mathrm{a}$ & 93.73a \\
\hline
\end{tabular}

${ }^{1}$ Factor A, temperaturas de fermentación, $1=20^{\circ} \mathrm{C}, 2=25^{\circ} \mathrm{C}, 3=30^{\circ} \mathrm{C}$; ${ }^{2}$ Factor $\mathrm{B}$, condiciones de fermentación, $1=$ =abierta (no airlock), $2=$ cerrada; ${ }^{3}$ Rendimiento de etanol (g etanol/g sólidos solubles); ${ }^{4} \mathrm{Glicerol}$; ${ }^{5}$ Fermentabilidad de maltotriosa (\%, inicial-final); ${ }^{6}$ Fermentabilidad de carbohidratos fermentables totales $(\%$, inicial-final). Medias con diferente letra entre tratamientos implican diferencia significativa (Tukey, $a=0.05$ ). 
by digital density meter, $-9 \mathrm{~B}$ Preparation of wort for color determination, -12 Free amino nitrogen, -13 viscosity, -14B Fermentable saccharides by High performance liquid chromatography, -17 Protein in unhopped wort by spectrophotometry. The Society, St. Paul, MN.

Aslankoohi, E., Rezaei, M.N., Vervoort, Y., Courtin, C.M., and Verstrepen, K.J. 2015. Glycerol production by fermenting yeast cells is essential for optimal bread dough fermentation. PLoS ONE 10(3): e0119364. DOI 10.1371/ journal.pone.0119364

Aylott, R. 2014. Whisky analysis. En: Whisky: Technology, production and marketing, 2nd. Edition. I. Russell y G. Stewart (ed.), pp 243-270. Academic Press, UK.

Bathgate, G.N. 2016. A review of malting and malt processing for whisky distillation. Journal of the Institute of Brewing 122: 197-211.

Bringhurst, T.A. y Brosnan, J. 2014. Scotch whisky: Raw material selection and processing. En: Whisky: Technology, production and marketing, 2nd. Edition. I. Russell y G. Stewart (ed.), pp 49-122. Academic Press, UK.

Cronwright, G.R., Rohwer, J.M., and Prior, B.A. 2002. Metabolic Control Analysis of Glycerol Synthesis in Saccharomyces cerevisiae. Applied and Environmental Microbiology 68(9): 4448-4456.

Cutaia, A.J. 2007. Estimation of yeast mass increase in production brewing fermentations by calculation of carbohydrate utilization. Journal of the American Society of Brewing Chemists 65(3): 166-171.

Evans, E.D., Collins, H., Eglington, J., and Wilhelmson, A. 2005. Assessing the impact of the level of diastatic power enzymes and their thermostability on the hydrolysis of starch during wort production to predict malt fermentability. Journal of the American Society of Brewing Chemists 63(4): 185-198.

Evans, E.D., and Fox, G.P. 2017. Comparison of diastatic power enzyme release and persistence during modified Institute of Brewing $65^{\circ} \mathrm{C}$ and Congress programmed mashes. Journal of the American Society of Brewing Chemists 75(4): 302-311.

Evans, E.D., Goldsmith, M., Dambergs, R., and Nischwitz, R. 2011. A comprehensive revaluation of small-scale Congress Mash protocol parameters for determining extract and fermentability. Journal of the American Society of Brewing Chemists 69(1): 13-27.

Evans, E.D., Goldsmith, M., Redd, K.S., Nischwitz, R., and Lentini, A. 2012. Impact of mashing conditions on extract, its fermentability, and the levels of wort free amino nitrogen (FAN), $\beta$-Glucan, and Lipids. Journal of the American Society of Brewing Chemists 70(1): 39-49.

Evans, E.D., Li, C., and Eglinton, J.K. 2010. The properties and genetics of barley malt starch degrading enzymes. En: Genetics and improvement of barley malt quality. G. Zhang y C. Li (ed.), pp 143-189. Zhejiang University Press, Hangzhou, China.

Fukuyo, S., and Myojo, Y. 2014. Japanese whisky. En: Whisky: Technology, production and marketing, 2nd. Edition. I. Russell y G. Stewart (ed.), pp 17-26. Academic Press, UK.

Gibson, T.S., Solah, V., Glennie Holmes, M.R., and Taylor, H.R. 1995. Diastatic power in malted barley: Contributions of malt parameters to its development and the potential of barley grain beta-amylase to predict malt diastatic power. Journal of the Institute of Brewing 101:277-280.
Henson, C.A., Duke, S.H., and Vinje, M.A. 2014. A comparison of barley malt amylolytic enzyme thermostabilities and wort sugars produced during mashing. Journal of the American Society of Brewing Chemists 72(1): 51-65.

Hill, A., and Stewart, G.G. 2019. Free amino nitrogen in brewing. Fermentation 5,22; doi:10.3390/fermentation5010022

Huerta, Z.R., Zamora, D.M., Solano, H.S., y López, C.M.L. 2014. Friabilidad de malta y predicción de calidad en el mejoramiento genético de cebada maltera (Hordeum vulgare L.). Revista Mexicana de Ciencias Agrícolas 5(4): 577 590.

Jin, Y.-L., Speers, R.A., and Paulson, A.T. 2004. Effect of $\beta$-glucans and process conditions on the membrane filtration performance of beer. Journal of the American Society of Brewing Chemists 62(3): 117-124.

Kwak, H.S., Seo, J.S., Hur, Y., Shim, H.-S., Lee, Y., Kim, M., and Jeong, Y. 2015. Influence of yeast strains on the physicochemical characteristics, methanol and acetaldehyde profiles and volatile compounds for Korean rice distilled spirit. Journal of the Institute of Brewing 121: 574-580.

Li, H., Han, X., Liu, F., Kun-Farkas, G., and Kiss, Z. 2015. Simple HPLC method for determining the glycerol content of beer. Journal of the American Society of Brewing Chemists 73(4): 314-317.

Lyons, T.P. 2003. Production of Scotch and Irish whiskies: their history and evolution. En:The alcohol textbook, 4th. Edition. K.A. Jacques, T.P. Lyons, y D.R. Kelsall (ed.), pp 194-222. Nottingham University Press, UK.

Lyons, T.P. 2014. North American whiskies: A story of evolution, experience, and an ongoing entrepreneurial spirit. En: Whisky: Technology, production and marketing, 2nd. Edition. I. Russell y G. Stewart (ed.), pp 39-48. Academic Press, UK.

Miedl, M., García, M.A., and Bamforth, C.W. 2005. Haze formation in model beer systems. Journal of Agricultural and Food Chemistry 53(26): 10161-10165.

Muller, R. 1991. The effects of mashing temperature and mash thickness on wort carbohydrate composition. Journal of the Institute of Brewing 97:85-92.

Muralikrishna, G., and Nirmala, M. 2005. Cereal a-amylases - an overview. Carbohydrate Polymers 60: 163-173.

Nevoigt, E., and Stahl, U. 1997. Osmoregulation and glycerol metabolism in the yeast Saccharomyces cerevisiae. FEMS Microbiology Reviews 21: 231-241.

Nicol, D.A. 2014. Batch distillation. En: Whisky: Technology, production and marketing, 2nd. Edition. I. Russell y G. Stewart (ed.), pp 155-178. Academic Press, UK.

Ohimain, E.I. 2016. Methanol contamination in traditionally fermented alcoholic beverages: the microbial dimension. SpringerPlus 5:1607. DOI 10.1186/s40064-016-3303-1

Qi, J.C., Zhang, G.P. y Zhou, M.X. 2006. Protein and hordein content in barley seeds as affected by nitrogen level and their relationship to beta-amylase activity. Journal of Cereal Science 43: 102-107.

Quinn, D. 2014. Irish whiskey. En:Whisky:Technology, production and marketing, 2nd. Edition. I. Russell y G. Stewart (ed.), pp 7-16. Academic Press, UK.

Rosenfeld, E., Beauvoit, B., Blondin, B., and Salmon, J.-M. 2003. Oxygen consumption by anaerobic Saccharomyces cerevisiae under enological conditions: Effect on fermentation kinetics. Applied and Environmental Microbiology 69(1): 113-121. 
Russell, I., and Stewart, G. 2014. Distilling yeast and fermentation. En: Whisky: Technology, production and marketing, 2nd. Edition. I. Russell y G. Stewart (ed.), pp 123-146. Academic Press, UK.

Sadosky, P., Schwarz, P.B., and Horsley, R. 2002. Effect of arabinoxylans, $\beta$-glucans, and dextrins on the viscosity and membrane filterability of a beer model solution. Journal of the American Society of Brewing Chemists 60(4): 153-162.

Scanes, K.T., Hohmann, S., and Priori, B.A. 1998. Glycerol production by the yeast Saccharomyces cerevisiae and its relevance to wine: A review. South African Journal of Enology and Viticulture 19(1): 17-24.

Schwarz, P.B., Li, Y., Barr, J., y Horsley, R.D. 2007. Effect of operational parameters on the determination of laboratory extract and associated wort quality factors. Journal of the American Society of Brewing Chemists 65(4): 219-228.

Statistical Analysis System (SAS). 2011. SAS Software. SAS Institute Inc., Cary, NC, USA.

Stenholm, K., and Home, S. 1999. A new approach to limit dextrinase and its role in mashing. Journal of the Institute of Brewing 105(4): 205-210.

Stewart, G.G. 2009. The Horace Brown medal lecture: forty years of brewing research. Journal of the Institute of Brewing 115(1): 3-29.
Stewart, G.G. 2010. High-gravity brewing and distilling - Past experiences and future prospects. Journal of the American Society of Brewing Chemists 68(1): 1-9.

Stewart, G.G., Hill, A.E., and Russell, I. 2013. 125th Anniversary review: Developments in brewing and distilling yeast strains. Journal of the Institute of Brewing 119: 202-220.

Vanderhaegen, B., Neven, H., Verachtert, H., and Derdelinckx, G. 2006. The chemistry of beer aging - A critical review. Food Chemistry 95: 357-381.

Wei, K., Dai, F., Wu, F.y Zhang, G. 2009. The variation of $\beta$-amylase activity and protein fractions in barley grains as affected by genotypes and post-anthesis temperatures. Journal of the Institute of Brewing 115(3): 208-213.

Willaert, R. 2007. The beer brewing process: Wort production and beer fermentation. En: Handbook of food products manufacturing. Y.H. Hui (ed.), pp 443-506. John Wiley \& Sons, NJ.

Wilson, N. 2014. Contamination: Bacteria and wild yeasts in a whisky fermentation. En: Whisky: Technology, production and marketing, 2nd. Edition. I. Russell y G. Stewart (ed.), pp 147-154. Academic Press, UK.

Younis, O.S., and Stewart, G.G. 1999. Effect of malt wort, veryhigh-gravity malt wort, and very-high-gravity adjunct wort on volatile production in Saccharomyces cerevisiae. Journal of the American Society of Brewing Chemists 57(2): 39-45. 